\title{
Different value of coronary calcium score to predict obstructive coronary artery disease in patients with and without moderate chronic kidney disease
}

\author{
K. H. Yiu • F. R. de Graaf - J. E. van Velzen • \\ N. A. Marsan - C. J. Roos • M. K. de Bie • H. F. Tse • \\ E. E. van der Wall • M. J. Schalij • J. J. Bax • \\ J. D. Schuijf • J. W. Jukema \\ Published online: 12 April 2013 \\ (C) The Author(s) 2013. This article is published with open access at Springerlink.com
}

\begin{abstract}
Purpose The coronary calcium score (CCS) predicts significant coronary artery disease $(\mathrm{CAD})$ in the general population. While moderate chronic kidney disease (CKD) is associated with high CCS, the use of CCS to predict significant CAD in these patients is unknown.

Methods A total of 704 patients underwent computed tomography coronary angiography for the assessment of CCS and CAD. Sixty-nine (10\%) patients had moderate CKD, defined by an estimated glomerular filtration rate (eGFR) between 30 and $59 \mathrm{~mL} / \mathrm{min} / 1.73 \mathrm{~m}^{2}$, and the remaining patients were considered to be without significant CKD (eGFR $\geq 60 \mathrm{~mL} / \mathrm{min} / 1.73 \mathrm{~m}^{2}$ ).

Results Patients with moderate CKD were older, had a higher CCS, and a higher prevalence of obstructive CAD than patients without significant CKD. Receiver-operator curve analysis showed that CCS predicted the presence of obstructive CAD in both patients with moderate CKD and those without significant CKD. In patients with moderate CKD, the optimal cut-off value of CCS to diagnose obstructive CAD was 140 (sensitivity $73 \%$
\end{abstract}

K. H. Yiu • F. R. de Graaf • J. E. van Velzen • N. A. Marsan •

C. J. Roos $\cdot$ M. K. de Bie $\cdot$ E. E. van der Wall $\cdot$ M. J. Schalij •

J. J. Bax $\cdot$ J. D. Schuijf $\cdot$ J. W. Jukema $(\bowtie)$

Department of Cardiology, Leiden University Medical Center,

Albinusdreef 2, 2333 ZA Leiden, the Netherlands

e-mail: J.W.jukema@lumc.nl

K. H. Yiu • N. A. Marsan • E. E. van der Wall • M. J. Schalij •

J. W. Jukema

The Interuniversity Cardiology Institute of the Netherlands,

Utrecht, the Netherlands

K. H. Yiu $\cdot$ H. F. Tse

Cardiology Division, Department of Medicine,

University of Hong Kong, Hong Kong, People's Republic of China and specificity of $70 \%$ ), and is 2.8 fold higher than in patients without significant CKD (cut-off value $=50$; sensitivity $75 \%$ and specificity $75 \%$ ).

Conclusion The present results demonstrate that CCS can predict obstructive CAD in patients with moderate CKD, although the optimal cut-off value is higher than in patients without significant CKD.

Keywords Coronary calcification $\cdot$ Chronic kidney disease Coronary artery disease

\section{Introduction}

In patients with end-stage chronic kidney disease (CKD) requiring dialysis, cardiovascular mortality is 5 times higher than in the general population [1]. Furthermore, studies have demonstrated that even early stages of $\mathrm{CKD}$, which are more prevalent than end-stage CKD requiring dialysis [2], are associated with elevated risk of cardiovascular disease and mortality [3, 4]. Therefore, accurate detection of cardiovascular disease in patients with early stages of CKD is important for accurate risk stratification.

The coronary calcium score (CCS) measured by computed tomography can predict the presence of significant coronary artery disease (CAD) in the general population [5-10]. While end-stage CKD is associated with elevated CCS as compared with the general population, several reports have demonstrated conflicting results in the correlation between CCS and the presence of significant CAD [10-13]. Moreover, the role of CCS to detect CAD in patients with moderate $\mathrm{CKD}$, who are at risk for future cardiovascular events, is unknown. Therefore the aim of the current study was to evaluate the predictive role of CCS for diagnosing 
CAD by computed tomography coronary angiography (CTA) in patients with moderate CKD compared with patients without significant CKD.

\section{Methods}

Study population

The study population consisted of 704 patients who underwent CCS and CTA assessment for suspected CAD. Patients were enrolled at the Leiden University Medical Center. Exclusion criteria included cardiac arrhythmias, severe renal insufficiency (defined as an eGFR $<30 \mathrm{~mL} / \mathrm{min} / \mathrm{m}^{2}$ ), known hypersensitivity to iodine contrast media and pregnancy.

Classification of moderate chronic kidney disease

Serum creatinine levels were used to assess the eGFR calculated with the Modified Diet in Renal Disease equation [14]. In order to prevent contrast-induced renal dysfunction affecting the analysis, only serum creatinine levels obtained prior to the CTA examination (up to 180 days prior to CTA) were used. Of note, patients with suspected acute renal failure (defined by an increase in serum creatinine of $\geq 0.5 \mathrm{mg} / \mathrm{dl}$ in $<2$ weeks or an increase of $>20 \%$ over baseline if baseline serum creatinine was $\geq 2.5 \mathrm{mg} / \mathrm{dl}$ ) were excluded.

Patients were stratified into those with moderate CKD and those without significant CKD. The definition of moderate CKD was based on the recommendation from the National Kidney Foundation [15] using a value of eGFR between 30 and $59 \mathrm{~mL} / \mathrm{min} / 1.73 \mathrm{~m}^{2}$. Patients with eGFR $\geq 60 \mathrm{~mL} / \mathrm{min} / 1.73 \mathrm{~m}^{2}$ were considered to have no significant $\mathrm{CKD}$. The mean duration between renal function assessment and CTA was $37 \pm 14$ days.

Computed tomography coronary angiography protocol

Examinations of CCS and CTA were performed using a 64row (Aquillion64, Toshiba Medical Systems, Tokyo Japan) computed tomography scanner. Descriptions of scan parameters for CCS and CTA assessment have been published previously $[16,17]$.

Data analysis

Post-processing of the CCS and CTA was performed on dedicated workstations (Vitrea2, Vital Images, Minneapolis, Minnesota, USA). The CCS was calculated using the Agatston method and patients were stratified as CCS 0, CCS 1 to 399 and $\mathrm{CCS} \geq 400$. Coronary anatomy was assessed in a standardised method by dividing the coronary arteries into 17 segments [18]. All CTA were interpreted by two experienced cardiologists blinded to the results of the CCS and eGFR. Classification of CTA results was made between nonobstructive and obstructive CAD using a luminal narrowing $\geq 50 \%$ as a threshold for obstructive CAD lesions. In addition, the number of segments (among the 17 segments) and vessels (among the 3 coronaries) involved in each category of CAD (presence of $\mathrm{CAD}$, non-obstructive $\mathrm{CAD}$ and obstructive CAD) was measured.

\section{Statistical analysis}

Continuous variables are presented as mean \pm standard deviation and compared using either Student's $t$ or Wilcoxon's rank-sum test as appropriate. Categorical data are presented as frequencies and percentages and compared using the chi-square or Fisher's exact test. Receiver-operator characteristic (ROC) curve analysis was performed to determine the value of CCS in diagnosing obstructive CAD. The optimal cut-off value was defined as the maximised value for the sum of sensitivity and specificity. In addition, the CCS values to diagnose obstructive CAD for a predefined 1) sensitivity of $80 \%$ and 2) specificity of $80 \%$ in patients with moderate $\mathrm{CKD}$ and without significant $\mathrm{CKD}$ were evaluated. All statistical analyses were performed using the statistical package SPSS for Windows (Version 15.0, SPSS, Chicago, USA). A $p$ value $<0.05$ was considered to be statistically significant.

\section{Results}

Baseline clinical characteristics

Baseline characteristics of the patient population (704 patients who underwent CCS and CTA) are shown in Table 1. The average age of all patients was $56 \pm 12$ years and $55 \%$ of patients were male. The mean eGFR for all patients was $84.7 \pm 21.5 \mathrm{~mL} / \mathrm{min} / 1.73 \mathrm{~m}^{2}$. Patients with moderate CKD were more likely to be older, obese and to have a history of hypertension while patients without significant CKD were more likely to be a smoker.

Coronary calcium score

The mean and median CCS of the whole population was 274 and19 respectively. Coronary calcium was absent in $36 \%$ patients, a CCS of 1 to 399 was present in $46 \%$ patients and $\mathrm{CCS} \geq 400$ in $18 \%$ patients. The mean and median CCS of patients with moderate CKD were higher than in patients without significant CKD. Moreover, patients 
Table 1 Baseline demographics on all patients, patients with moderate chronic kidney disease (CKD) and patients without significant CKD

$B M I$ body mass index; $C A D$ coronary artery disease; $M I$ myocardial infarction; $P C I$ percutaneous coronary intervention

\begin{tabular}{lllll}
\hline & All $(n=704)$ & $\begin{array}{l}\text { Moderate } \\
\text { CKD }(n=69)\end{array}$ & $\begin{array}{l}\text { No significant } \\
\text { CKD }(n=635)\end{array}$ & $p$ value \\
\hline $\begin{array}{llll}\text { Age (years) } \\
\text { Male gender (\%) }\end{array}$ & $56 \pm 12$ & $62 \pm 12$ & $55 \pm 12$ & $<0.01$ \\
Risk factors (\%) & $385(54.7)$ & $39(56.5)$ & $346(54.5)$ & 0.80 \\
- Diabetes & & & & \\
- Hypertension & $245(34.8)$ & $27(39.1)$ & $218(34.3)$ & 0.43 \\
- Hypercholesterolaemia & $329(46.7)$ & $43(62.3)$ & $286(45)$ & $<0.01$ \\
- Family history of CAD & $281(39.9)$ & $35(50.7)$ & $246(38.7)$ & 0.07 \\
- Smoking & $331(44.2)$ & $26(37.7)$ & $285(44.9)$ & 0.31 \\
- Obesity BMI $>30 \mathrm{~kg} / \mathrm{m}^{2}$ & $149(21.2)$ & $7(10.1)$ & $142(22.4)$ & 0.02 \\
Previous MI (\%) & $150(21.3)$ & $24(34.8)$ & $126(19.8)$ & $<0.01$ \\
Previous PCI (\%) & $41(5.8)$ & $4(5.8)$ & $37(5.8)$ & 1.00 \\
\hline
\end{tabular}

with moderate CKD had a higher prevalence of CCS 1-399 and $\mathrm{CCS} \geq 400$ than patients without significant CKD (Table 2).

Computed tomography coronary angiography results

Presence of CAD was detected in $64 \%$ of patients including non-obstructive CAD in $33 \%$ and obstructive CAD in $31 \%$ of patients. Patients with moderate $\mathrm{CKD}$ had a higher prevalence of CAD, increased number of segments and vessels with CAD as compared with patients without significant CKD (Table 2). In addition, patients with moderate CKD had a higher prevalence of non-obstructive CAD although this observation did not reach statistical significance ( $p=$ 0.059). Nevertheless, the number of segments and vessels involved with non-obstructive CAD was higher in patients with moderate CKD. In contrast, the prevalence of obstructive
$\mathrm{CAD}$ and the number of segments and vessels with obstructive CAD was similar between patients with moderate CKD and those without significant CKD.

CTA results in relation to coronary calcium score

The results of CTA in relation to CCS subgroups in patients with moderate $\mathrm{CKD}$ and patients without significant CKD are shown in Table 3. Among the two groups of patients with moderate CKD and patients without significant CKD, the prevalence of the presence of CAD, non-obstructive CAD and obstructive CAD was significantly higher in subgroups of patients with a higher CCS. Moreover, the number of segments and vessels involved with the presence of $\mathrm{CAD}$, non-obstructive CAD and obstructive CAD increased in subgroups of patients with higher CCS.
Table 2 Coronary calcium score (CCS) and computed tomography coronary angiography results in patients with moderate CKD and without significant CKD

\begin{tabular}{lllll}
\hline & $\begin{array}{l}\text { All patients } \\
(n=704)\end{array}$ & $\begin{array}{l}\text { Moderate } \\
\text { CKD }(n=69)\end{array}$ & $\begin{array}{l}\text { No significant } \\
\text { CKD }(n=635)\end{array}$ & $p$ value \\
\hline Mean CCS & $273 \pm 760$ & $592 \pm 1074$ & $265 \pm 712$ & 0.02 \\
Median CCS (Interquatile range) & $19(0,265)$ & $129(22,746)$ & $14(0,216)$ & \\
- CCS 0 (\%) & $255(36.2)$ & $11(15.9)$ & $244(38.4)$ & 0.01 \\
- CCS 1-399 (\%) & $320(45.5)$ & $36(52.2)$ & $284(44.7)$ & \\
- CCS $>400(\%)$ & $129(18.3)$ & $22(31.9)$ & $107(16.9)$ & \\
Presence of CAD (\%) & $451(64.1)$ & $56(81.2)$ & $395(62.2)$ & $<0.01$ \\
- Number of segments & $3.5 \pm 3.8$ & $4.8 \pm 4.0$ & $3.3 \pm 3.8$ & $<0.01$ \\
- Number of vessels & $1.7 \pm 1.3$ & $2.3 \pm 1.1$ & $1.7 \pm 1.3$ & $<0.01$ \\
Presence of non-obstructive CAD (\%) & $232(33.0)$ & $30(43.5)$ & $202(31.8)$ & 0.059 \\
- Number of segments & $2.8 \pm 3.1$ & $3.9 \pm 3.4$ & $2.6 \pm 3.1$ & $<0.01$ \\
- Number of vessels & $1.7 \pm 1.3$ & $2.2 \pm 1.1$ & $1.6 \pm 1.3$ & $<0.01$ \\
Presence of obstructive CAD (\%) & $219(31.1)$ & $26(37.7)$ & $193(30.4)$ & 0.22 \\
- Number of segments & $0.7 \pm 1.4$ & $1.0 \pm 1.7$ & $0.7 \pm 1.4$ & 0.20 \\
- Number of vessels & $0.5 \pm 0.9$ & $0.6 \pm 0.9$ & $0.5 \pm 0.9$ & 0.28 \\
\hline
\end{tabular}


Table 3 Results of computed tomography coronary angiography according to CCS subgroups in patients with moderate CKD and without significant CAD
Abbreviations as for Table 1

\begin{tabular}{lllll}
\hline & CCS $=0$ & CCS 1-399 & CCS $\geq 400$ & $p$ value \\
\hline Moderate CKD & & & & \\
Presence of CAD (\%) & $2(18.2)$ & $32(88.9)$ & $22(100)$ & $<0.01$ \\
- Number of segments & $0.4 \pm 0.9$ & $3.6 \pm 2.9$ & $9.1 \pm 2.6$ & $<0.01$ \\
- Number of vessels & $0.4 \pm 0.7$ & $2.5 \pm 0.8$ & $3.0 \pm 0.2$ & $<0.01$ \\
Presence of non-obstructive CAD (\%) & $1(9.1)$ & $22(61.1)$ & $7(31.8)$ & $<0.01$ \\
- Number of segments & $0.2 \pm 0.4$ & $3.1 \pm 2.6$ & $7.0 \pm 2.9$ & $<0.01$ \\
- Number of vessels & $0.3 \pm 0.5$ & $2.4 \pm 0.8$ & $2.9 \pm 0.4$ & $<0.01$ \\
Presence of obstructive CAD (\%) & $1(9.1)$ & $10(27.8)$ & $15(68.2)$ & $<0.01$ \\
- Number of segments & $0.2 \pm 0.6$ & $0.5 \pm 1.1$ & $2.1 \pm 2.3$ & $<0.01$ \\
- Number of vessels & $0.1 \pm 0.3$ & $1.3 \pm 1.1$ & $1.3 \pm 1.1$ & $<0.01$ \\
Without significant CAD & & & & $<0.01$ \\
Presence of CAD (\%) & $54(22.1)$ & $235(82.7)$ & $106(99.1)$ & $<0.01$ \\
- Number of segments & $0.6 \pm 1.4$ & $3.7 \pm 3.1$ & $8.7 \pm 3.1$ & $<0.01$ \\
- Number of vessels & $0.5 \pm 1.0$ & $2.2 \pm 1.0$ & $2.9 \pm 0.3$ & $<0.01$ \\
Presence of non-obstructive CAD (\%) & $37(15.2)$ & $137(8.2)$ & $28(26.2)$ & $<0.01$ \\
- Number of segments & $0.5 \pm 1.2$ & $3.0 \pm 2.6$ & $6.5 \pm 2.9$ & $<0.01$ \\
- Number of vessels & $0.5 \pm 0.9$ & $2.1 \pm 1.0$ & $2.8 \pm 0.5$ & $<0.01$ \\
Presence of obstructive CAD (\%) & $17(7.0)$ & $98(34.5)$ & $78(72.9)$ & $<0.01$ \\
- Number of segments & $0.1 \pm 0.4$ & $0.6 \pm 1.2$ & $2.2 \pm 2.2$ & $<0.01$ \\
- Number of vessels & $0.1 \pm 0.3$ & $0.5 \pm 0.8$ & $1.4 \pm 1.1$ & $<0.01$ \\
\hline
\end{tabular}

Prediction of obstructive coronary artery disease

To evaluate the predictive value of CCS for diagnosing obstructive CAD in patients with moderate CKD and patients without significant CKD, ROC curve analysis was performed. As shown in Fig. 1, CCS predicted the presence of obstructive CAD in both patients with moderate CKD (area under curve $0.78, p<0.01$ ) and patients without significant CKD (area

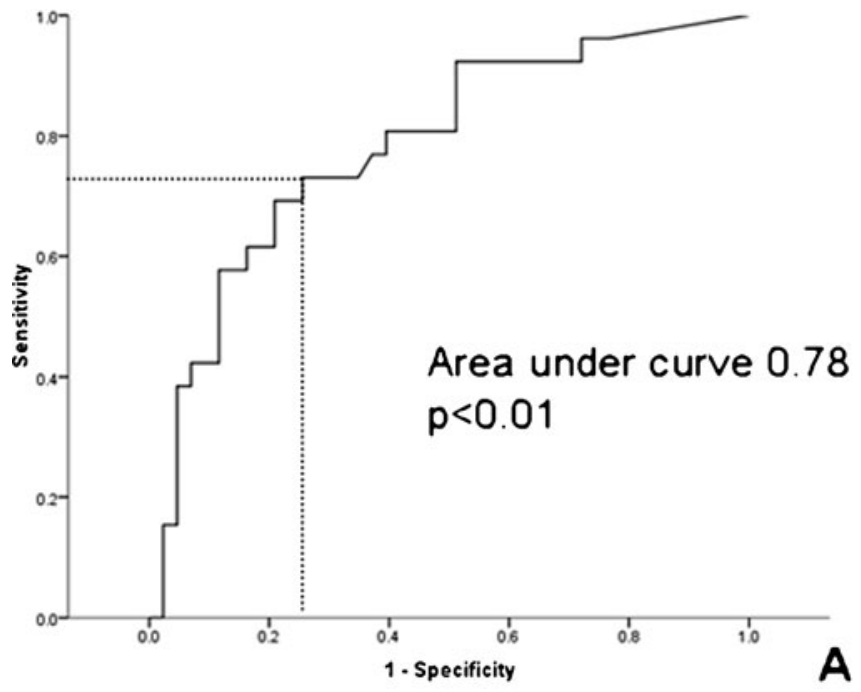

Fig. 1 Receiver-operator characteristics curve analysis of patients with moderate chronic kidney disease (CKD) (Panel a) and without significant CKD (Panel b). The optimal cut-off value of the coronary calcium score (CCS) to diagnose obstructive coronary artery disease under curve $0.82, p<0.01)$. The individual sensitivity and specificity of CCS to predict obstructive CAD in patients with moderate CKD and patients without significant CKD are shown in Fig. 2. In patients with moderate CKD, the optimal cut-off value of CCS to diagnose obstructive CAD was 140 (sensitivity $73 \%$ and specificity of $70 \%$ ). On the other hand, the optimal cut-off value of CCS for patients without significant $\mathrm{CKD}$ to diagnose obstructive CAD was 50 (sensitivity

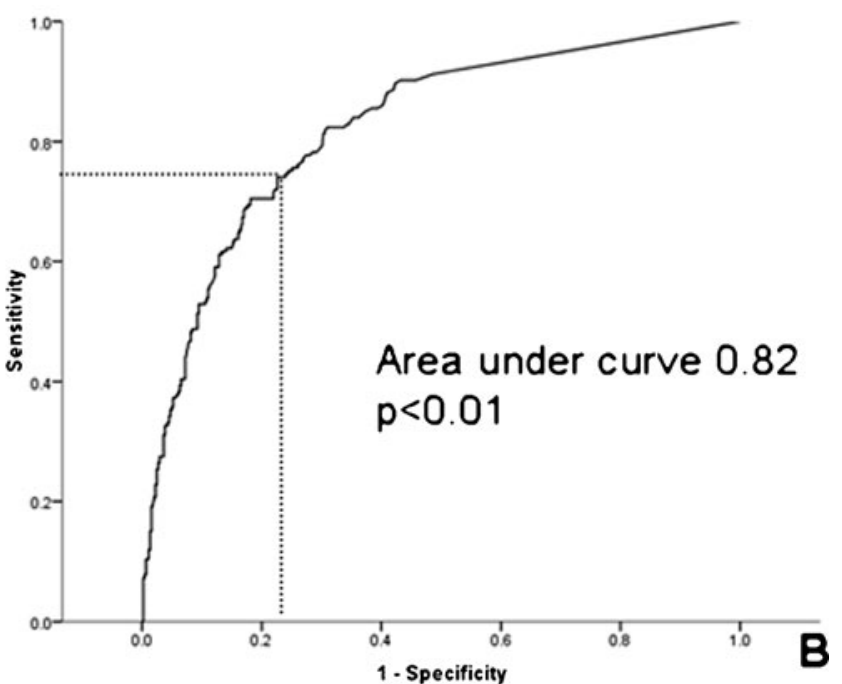

(CAD) in patients with moderate CKD is 140 (sensitivity $73 \%$ and specificity $70 \%$ ). The optimal cut-off value of CCS to diagnose obstructive CAD in patients without significant CKD is 50 (sensitivity $75 \%$ and specificity $75 \%$ ) 


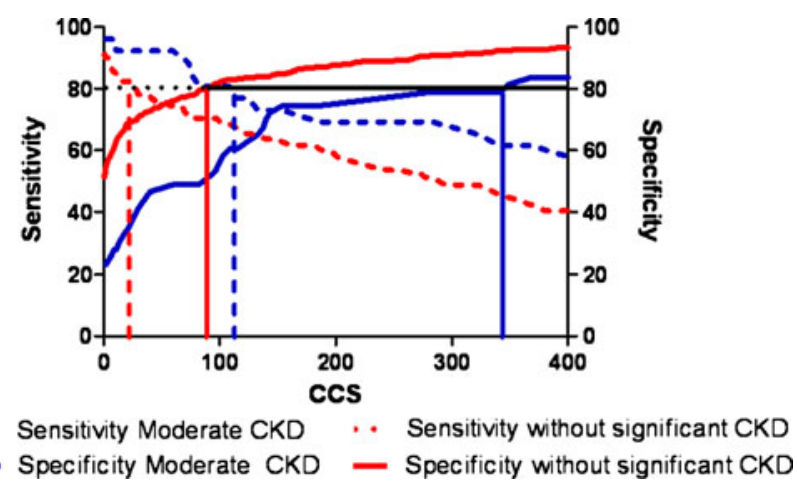

Fig. 2 Sensitivity (dotted lines) and specificity (solid lines) of coronary calcium score (CCS) to predict CAD in patients with moderate CKD (blue lines) and patients without significant CKD (red lines). The value of CCS is higher in patients with moderate CKD than in patients without significant CKD for a given sensitivity and specificity. Example: a predefined sensitivity and specificity of $80 \%$ (black lines) would require a higher CCS in patients with moderate CKD than patients without significant CKD (110 vs. 27 and 345 vs. 90, respectively)

$75 \%$ and specificity $75 \%$ ). To achieve a predefined sensitivity of $80 \%$ for diagnosing obstructive $\mathrm{CAD}$, the required value of CCS in patients with moderate CKD was higher than in patients without significant CKD (110 vs. 27, corresponding specificity $60 \%$ vs. $70 \%$ respectively). Similarly, a higher CCS was required to achieve a predefined specificity of $80 \%$ for diagnosing obstructive CAD in patients with moderate CKD than in patients without significant CKD (345 vs. 90, corresponding sensitivity $62 \%$ vs. $71 \%$ respectively).

\section{Discussion}

The present report demonstrates that patients with moderate CKD had a higher prevalence, more diffuse and greater extent of coronary calcium and CAD than patients without significant CKD. Moreover, CCS predicted the presence of obstructive CAD in both patients with moderate CKD and patients without significant CKD. Importantly, the optimal cut-off value of CCS for predicting the presence of obstructive CAD was higher in patients with moderate CKD than patients without significant CKD.

Recent studies using computed tomography demonstrated that patients with early stages of CKD had a higher prevalence of CCS and CAD than patients without CKD $[19,20]$. This is in concordance with the present results, demonstrating a higher prevalence, more diffuse and greater extent of coronary calcium and CAD in patients with moderate CKD than patients without significant CKD. Accordingly, these results provided further supporting evidence for the relationship between elevated cardiovascular risk and moderate $\mathrm{CKD}[3,4]$. Interestingly, the increased prevalence of CAD in patients with moderate CKD was mainly contributed by the presence of non-obstructive
CAD. The observation may partially be explained by the thickening and calcification in the media layer, instead of the intima layer, of the coronaries in patients with moderate $\mathrm{CKD}$, a phenomenon that is classically described as Mönckeberg's calcification or medial calcinosis [21, 22]. Therefore, in patients with moderate CKD, the elevated CCS located in the medial layer may be associated with non-obstructive coronary atherosclerosis, rather than significant luminal obstruction [23].

Although the coronary calcification and degree of CAD differed between patients with and without CKD, a close relation between CCS and presence of obstructive CAD has been shown in the general population $[24,25]$ as well as in patients with end-stage CKD [10-12]. The present results further showed that high CCS was associated with higher prevalence, more diffuse and greater extent of CAD in both patients with moderate CKD and patients without significant CKD.

In addition to the significant relation with $\mathrm{CAD}$, previous reports have demonstrated that the use of CCS can predict the presence of obstructive $\mathrm{CAD}$ in the general population $[6,7]$. Moreover, few studies have evaluated the predictive role of CCS for obstructive CAD in patients with end-stage CKD. In the report by Sharples evaluating 18 patients with CKD requiring dialysis, the presence of CCS was not correlated with obstructive CAD diagnosed by coronary angiogram [13]. Conversely, Fujimoto et al. demonstrated a CCS value of 1000 provided a sensitivity of $68 \%$ and specificity of $69 \%$ to diagnose obstructive CAD in 76 patients with $\mathrm{CKD}$ requiring dialysis [10]. In addition, Robinson and colleagues showed a CCS value of 400 provided a sensitivity of $86 \%$ and specificity of $83 \%$ in diagnosing obstructive CAD in 37 patients with CKD (17 of them requiring dialysis) [12]. While these studies mainly evaluated patients with end-stage CKD, none of them explored the role of CCS to predict obstructive $\mathrm{CAD}$ specifically in patients with moderate $\mathrm{CKD}$, which is more common in the general population [2]. Nonetheless, patients with moderate CKD have a greater cardiovascular risk than the general population, highlighting the need for appropriate strategies to detect obstructive CAD [3, 4]. The results of the present study have thus confirmed the predictive role of CCS in diagnosing obstructive CAD in patients with moderate CKD. However, the optimal cut-off value of CCS was 2.8 fold higher in patients with moderate CKD than patients without significant CKD. For the same sensitivity and specificity to diagnose obstructive $\mathrm{CAD}$, the value of CCS was consistently higher in patients with moderate CKD than patients without significant CKD (Fig. 2). The reason for this observation has not been fully elucidated, but may possibly be explained by the different morphology and distribution of coronary calcification in patients with and without CKD. Patients with CKD appear to have more diffuse calcified plaque burden that tends to be located in the media wall rather than protruding into the lumen [26]. Therefore, while the 
relation between elevated CCS and obstructive CAD existed in patients with moderate $\mathrm{CKD}$, the degree of this relationship is different as compared with patients without moderate CKD. Accordingly, the optimal cut-off value of CCS to predict the presence of obstructive CAD should be different in patients with moderate $\mathrm{CKD}$ as compared with patients without significant CKD. However, the exact value of CCS for this purpose requires further evaluation based on larger study populations.

\section{Limitation}

The present study consisted of patients with moderate CKD and the predictive role of CCS to diagnose obstructive CAD in patients with severe CKD $\left(\mathrm{eGFR}<30 \mathrm{~mL} / \mathrm{min} / 1.73 \mathrm{~m}^{2}\right)$ cannot be extrapolated. Similar to previous evaluations exploring the CTA results of patients with and without CKD, the present study confirmed that patients with moderate CKD were older as compared with patients without significant CKD [17]. However, due to the small population, the independent association between moderate CKD and high CCS/significant CAD could not be evaluated. Although CCS is proven to be a valuable tool to detect coronary calcification, it is unable to differentiate media calcification, which is common in patients with CKD, from intima calcification. Moreover, whether the use of computed tomography fractional flow reserve will provide additional diagnostic information in patients with CKD would require further studies [27].

\section{Conclusion}

The present study demonstrates that CCS predicted the presence of obstructive $\mathrm{CAD}$ in both patients with moderate $\mathrm{CKD}$ and those without significant CKD. More importantly, the optimal cut-off value and the value of CCS for the same sensitivity and specificity to diagnose obstructive CAD were higher in patients with moderate CKD than patients without significant $\mathrm{CKD}$. Therefore in the clinical setting, a higher cutoff value of CCS to predict the presence of obstructive CAD is required in patients with moderate $\mathrm{CKD}$ as compared with patients without significant CKD.

Funding Kai Hang Yiu is financially supported by a research grant from the Hong Kong Heart Foundation.

Fleur R. de Graaf was supported by the Dutch Technology Foundation STW (Utrecht, the Netherlands).

The Department of Cardiology of Leiden University Medical Center (The Netherlands) receives grants from Biotronik, BMS medical imaging, Boston Scientific, Edwards Lifesciences, GE Healthcare, Medtronic and St. Jude Medical.

JW Jukema receives research grants from and was a speaker at meetings sponsored by Astellas, AstraZeneca, Biotronic, Boston
Scientific, Bristol-Myers Squibb, Cordis, Daiichi Sankyo, Eli Lilly and Company, Medtronic, Merck-Schering Plough, Pfizer, Orbus Neich, Novartis, Roche, Servier, the Netherlands Heart Foundation, the Interuniversity Cardiology Institute of the Netherlands and the European Community Framework KP7 program.

The remaining authors: none.

Conflict of interests None declared.

Open Access This article is distributed under the terms of the Creative Commons Attribution License which permits any use, distribution, and reproduction in any medium, provided the original author(s) and the source are credited.

\section{References}

1. Sarnak MJ, Levey AS, Schoolwerth AC, et al. Kidney disease as a risk factor for development of cardiovascular disease: a statement from the american heart association councils on kidney in cardiovascular disease, high blood pressure research, clinical cardiology, and epidemiology and prevention. Circulation. 2003;108:2154-69.

2. National Kidney Foundation. K/DOQI clinical practice guidelines for chronic kidney disease: evaluation, classification, and stratification. Am J Kidney Dis. 2002;39:S1-266.

3. Go AS, Chertow GM, Fan D, et al. Chronic kidney disease and the risks of death, cardiovascular events, and hospitalization. N Engl J Med. 2004;351:1296-305.

4. Brosius III FC, Hostetter TH, Kelepouris E, et al. Detection of chronic kidney disease in patients with or at increased risk of cardiovascular disease: a science advisory from the American Heart Association Kidney and Cardiovascular Disease Council; the Councils on High Blood Pressure Research, Cardiovascular Disease in the Young, and Epidemiology and Prevention; and the Quality of Care and Outcomes Research Interdisciplinary Working Group: developed in collaboration with the National Kidney Foundation. Circulation. 2006;114:1083-7.

5. Budoff MJ, Achenbach S, Blumenthal RS, et al. Assessment of coronary artery disease by cardiac computed tomography: a scientific statement from the American Heart Association committee on cardiovascular imaging and intervention, council on cardiovascular radiology and intervention, and committee on cardiac imaging, council on clinical cardiology. Circulation. 2006;114:1761-91.

6. Budoff MJ, Diamond GA, Raggi P, et al. Continuous probabilistic prediction of angiographically significant coronary artery disease using electron beam tomography. Circulation. 2002;105:1791-6.

7. Haberl R, Becker A, Leber A, et al. Correlation of coronary calcification and angiographically documented stenoses in patients with suspected coronary artery disease: results of 1,764 patients. $\mathrm{J}$ Am Coll Cardiol. 2001;37:451-7.

8. Rijlaarsdam-Hermsen D, Kuijpers D, van Dijkman PR. Diagnostic and prognostic value of absence of coronary artery calcification in patients with stable chest symptoms. Neth Heart J. 2011;19:223-8.

9. Weustink AC, de Feyter PJ. The role of multi-slice computed tomography in stable angina management: a current perspective. Neth Heart J. 2011;19:336-43.

10. Fujimoto N, Iseki K, Tokuyama K, et al. Significance of coronary artery calcification score (CACS) for the detection of coronary artery disease (CAD) in chronic dialysis patients. Clin Chim Acta. 2006;367:98-102.

11. Haydar AA, Hujairi NM, Covic AA, et al. Coronary artery calcification is related to coronary atherosclerosis in chronic renal disease patients: a study comparing EBCT-generated coronary 
artery calcium scores and coronary angiography. Nephrol Dial Transplant. 2004;19:2307-12.

12. Robinson J, Tan AU, Wilensky RL, et al. Electron-beam computerized tomography correlates with coronary angiogram in chronic kidney disease patients. Am J Nephrol. 2007;27:247-52.

13. Sharples EJ, Pereira D, Summers S, et al. Coronary artery calcification measured with electron-beam computerized tomography correlates poorly with coronary artery angiography in dialysis patients. Am J Kidney Dis. 2004;43:313-9.

14. Levey AS, Coresh J, Greene T, et al. Using standardized serum creatinine values in the modification of diet in renal disease study equation for estimating glomerular filtration rate. Ann Intern Med. 2006;145:247-54.

15. Levey AS, Coresh J, Balk E, et al. National kidney foundation practice guidelines for chronic kidney disease: evaluation, classification, and stratification. Ann Intern Med. 2003;139:137-47.

16. van Werkhoven JM, de Boer SM, Schuijf JD, et al. Impact of clinical presentation and pretest likelihood on the relation between calcium score and computed tomographic coronary angiography. Am J Cardiol. 2010;106:1675-9.

17. Schuijf JD, Wijns W, Jukema JW, et al. Relationship between noninvasive coronary angiography with multi-slice computed tomography and myocardial perfusion imaging. J Am Coll Cardiol. 2006;48:2508-14.

18. Austen WG, Edwards JE, Frye RL, et al. A reporting system on patients evaluated for coronary artery disease. Report of the Ad Hoc Committee for Grading of Coronary Artery Disease, Council on Cardiovascular Surgery, American Heart Association. Circulation. $1975 ; 51: 5-40$
19. Cho I, Min HS, Chun EJ, et al. Coronary atherosclerosis detected by coronary $\mathrm{CT}$ angiography in asymptomatic subjects with early chronic kidney disease. Atherosclerosis. 2010;208:406-11.

20. Kestenbaum BR, Adeney KL, de Boer IH, et al. Incidence and progression of coronary calcification in chronic kidney disease: the Multi-Ethnic Study of Atherosclerosis. Kidney Int. 2009;76:9918.

21. Parfitt AM. Soft-tissue calcification in uremia. Arch Int Med. 1969;124:544-56.

22. Schiffrin EL, Lipman ML, Mann JF. Chronic kidney disease: effects on the cardiovascular system. Circulation. 1979;16:85-97.

23. Proudfoot D, Shanahan CM, Weissberg PL. Vascular calcification: new insights into an old problem. J Pathol. 1998; 185:1-3.

24. Budoff MJ, Georgiou D, Brody A, et al. Ultrafast computed tomography as a diagnostic modality in the detection of coronary artery disease: a multicenter study. Circulation. 1996;93: 898-904.

25. Guerci AD, Spadaro LA, Goodman KJ, et al. Comparison of electron beam computed tomography scanning and conventional risk factor assessment for the prediction of angiographic coronary artery disease. J Am Coll Cardiol. 1998;32:673-9.

26. Gross ML, Meyer HP, Ziebart H, et al. Calcification of coronary intima and media: immunohistochemistry, backscatter Imaging, and X-Ray analysis in renal and nonrenal patients. Clin J Am Soc Nephrol. 2007;2:121-34.

27. Meijs MF, Cramer MJ, El Aidi H, et al. CT fractional flow reserve: the next level in non-invasive cardiac imaging. Neth Heart J. $2012 ; 20: 410-8$. 